\title{
Estructura del consumidor, la frontera de producción y el marco de bienestar creado por la interacción del consumidor, productor y el mercado vinculado a la oferta $y$ demanda de medicamentos'
}

\author{
Jahir A. Gutiérrez O.² \\ Rubén D. Manrique H. ${ }^{3}$
}

doi:10.11144/Javeriana.ie 18-2.ecfp

Cómo citar: Gutiérrez JÁ, Manrique RD. Estructura del consumidor, la frontera de producción y el marco de bienestar creado por la interacción del consumidor, productor y el mercado vinculado a la oferta y demanda de medicamentos. Investig Enferm. Imagen Desarr. 2016;18(1): 153-173. doi:10.11144/Javeriana.ie18-2.ecfp

1. Este artículo es producto del proyecto de investigación Estructura de las empresas y la industria químico-farmacéutica frente a la economía de la salud y la geopolitica de los medicamentos: una revisión internacional y desarrollo en el caso colombiano. Recibido: 7 de noviembre de 2014. Aceptado: 17 de julio de 2015.

2. PhD en Administración Pública, Atlantic International University (AIU), Honolulú, Estados Unidos. Magíster en Desarrollo, Universidad Pontificia Bolivariana, Medellin, Colombia. Economista de la Universidad de Medellin, Colombia. Investigador y Líder del Grupo de Gestión Empresarial del CES, Facultad de Ciencias Administrativas, Universidad CES y Universidad del Rosario, Medellin, Colombia. Correo electrónico: jagogutierrez@gmail.com

3. PhD en Epidemiología, Universidad de Antioquia, Medellín, Colombia. Magíster en Epidemiologia, Universidad de Antioquia. Especialista en Altas Técnicas del Laboratorio Forense. Químico farmaceuta de la Universidad de Antioquia. Investigador del Grupo de Investigación ICIF-CES. Director de la Unidad de Gestión del Conocimiento de la Universidad CES en Medellín. Correo electrónico: rmanrique@ces.edu.co 


\title{
Resumen
}

Introducción: La sustentación del sector farmacéutico desde la economía representa un reto para su consideración, respecto al papel que desempeñan los actores inmiscuidos en el mercado de los medicamentos. Objetivo: Caracterizar la fundamentación económica del sector, la estructura del consumidor, la frontera de producción y el marco de bienestar creado por la interacción del consumidor, el productor y el mercado vinculado a la oferta y demanda de medicamentos. Metodologia: El análisis institucional funge como herramienta metodológica para allanar la composición de cada uno de los actores relacionados desde la combinación económica, jurídica y social, cuya máxima se encuentra en el acceso, disposición y oferta general de medicamentos. Desarrollo: La diversificación de agentes económicos, particularmente empresariales, financieros e industriales en el sector farmacéutico, ha dejado por fuera de la discusión a los consumidores, pacientes o usuarios. Conclusiones: El sector farmacéutico internacional está en mora de reorientar el perfil económico que lo ha caracterizado, en un campo en el que los medicamentos hacen las veces de bienes meritorios.

Palabras clave: economía farmacéutica; economía de la salud; industria farmacéutica; laboratorios; sector de atención en salud.

\section{Structure of Consumer, the Frontier of Production and Part of Welfare Created by the Interaction of Consumer, Producer and the Market Linked to the Supply and Demand for Drugs}

\begin{abstract}
Introduction: The bearing capacity of the pharmaceutical sector from the economy presents a challenge for its consideration, with regard to the role played by the actors involved in the drugs market. Aim: To characterize the economic foundation of the sector, the structure of the consumer, production frontier and the framework of welfare created by the interaction of the consumer, the producer and the market linked to the supply and demand of drugs. Methodology: Institutional analysis serves as a methodological tool to smooth out the composition of each of the actors since the economic, legal and social combination whose maximum is found in access, provision and general supply of drugs. Development: The diversification of economic, particularly business, financial and industrial agents in the pharmaceutical sector has left out of the discussion to consumers, patients and users. Conclusions: The international pharmaceutical industry is in arrears of reorienting the economic profile that has characterized, in a field in which drugs make meritorious goods as.
\end{abstract}

Keywords: pharmaceutical economy; health economics; pharmaceutical industry; laboratories; health care sector 


\section{Estrutura do consumidor, a fronteira de produção e parte do bem-estar criado pela interação do consumidor, o produtor e o mercado ligado à oferta e a procura de medicação}

\section{Resumo}

Introdução: A capacidade de rolamento do sector farmacêutico da economia é um desafio para a sua consideração, no que se refere o papel desempenhado pelos atores envolvidos no mercado de drogas. Objetivo: Caracterizar a base económica do sector, a estrutura do consumidor, fronteira de produção e a estrutura de bem-estar criado pela interação do consumidor e produtor o mercado ligado à oferta e a procura de drogas. Metodologia: Análise institucional serve como uma ferramenta metodológica para suavizar a composição de cada um dos atores, desde a combinação econômica, jurídica e social, cujo máximo é encontrada no acesso, disponibilização e fornecimento geral de drogas. Desenvolvimento: A diversificação dos agentes económicos, particularmente negócios, financeiros e industriais do setor farmacêutico, deixou fora da discussão para os consumidores, pacientes e usuários. Conclusões: A indústria farmacêutica internacional está em atraso de reorientar o perfil econômico que caracterizou, em um campo em que drogas fazem bens meritórios como.

Palavras chave: economia farmacêutica; economia da saúde; indústria farmacêutica; laboratórios; sector de cuidados de saúde. 


\section{Introducción}

La microeconomía expone la estructura del consumidor, del productor y del mercado de cara al equilibrio general, fundamento básico del análisis económico. En cuanto al consumidor, la relación precio-ingresos con respecto a la demanda y oferta de medicamentos no encuentra el punto de equilibrio. En cuanto al productor, la relación entre los costos, los excedentes y la producción no es tan expedita como se cree y, por ende, permite la concentración empresarial e impide la incubación de firmas centradas en la actividad, y no solo en el negocio.

El comportamiento del consumidor referido a la capacidad económica que tienen los clientes, pacientes o usuarios para adquirir los productos relacionados en dichas industrias no logra evidenciarse plenamente. Es de generalizado conocimiento que los precios de los productos farmacéuticos no logran adquirirse por vía ingresos o renta como debería de esperarse; a su vez, tampoco es claro el logro o maximización de la salud por cuenta de los medicamentos ofrecidos.

La conducta del productor caracterizada por la estructura de mercados a la cual atiende $u$ obedece expone una serie de empresas de carácter multinacional y transnacional, cuyo interés es ubicarse en el mercado de dominio de marcas, patentes y registros. De manera paralela, permanecen empresas de menor alcance y extensión, que atendiendo diversos segmentos hacen hincapié en su capacidad de atender a la población que requiere medicamentos a menor costo que las primeras.

La teoría de la firma, que hace las veces de análisis institucional, expone el balance que deben tener las empresas en cuanto equilibrio económico como el social. Si bien, las empresas del sector farmacéutico, laboratorios y multinacionales deben dar cuenta, es claro que su motivación está arraigada a las expectativas de ganancias o excedentes financieros, pero en poco o nada interesadas en evaluar el impacto de sus productos en diferentes campos.

El propósito de este artículo es profundizar sobre los elementos que engloban los fundamentos económicos de las empresas e industrias químico-farmacéuticas; la conducta del consumidor de productos farmacéuticos; la estructura empresarial de los productores farmacéuticos y de laboratorios químicos, y la estructura de equilibrio de mercados que exige tanto la conducta del consumidor de medicamentos como las empresas ubicadas en el sector.

\section{Fundamentos económicos de las empresas e industrias químico-farmacéuticas}

Es importante que se analice el alcance del fundamento económico a la hora de explicar la teoría del consumidor, el productor y el mercado del sector de medicamentos. Es un sector particular, dada la naturaleza de los bienes ofertados o producidos en el mercado que deben tomar un tiempo para su disposición o son escasos. Por ello, debe observarse la impronta que genera la teoría del consumidor frente a la conducta del comprador o usuario de medicamentos: "El marco teórico conceptual adoptado es el que entiende que 
los medicamentos se diferencian de la mayoría de las mercancías y servicios que el consumidor adquiere en el mercado" (1).

La economía analiza el comportamiento del consumidor, los empresarios y los mercados cuando se cuenta con información completa y plena, si existen referencias sobre el comportamiento del consumidor en materia de acceso e impacto de los medicamentos. A su vez, si el mercado ha logrado generar las condiciones para que el componente de medicamentos sea catalogado como parte de la economía de la salud. "La evaluación farmacoeconomía [sic] trata de ser una ayuda para mejorar la asignación de recursos económicos y las decisiones sobre la implementación de intervenciones sanitarias" (2).

El contenido de análisis en materia de medicamentos con respecto al consumidor gira en torno a las condiciones económicas y de acceso que tiene este en los medicamentos. No obstante, para el consumidor, el cliente o el usuario de los medicamentos, estos representan bienes hedónicos o, por lo menos, que contribuyen a mejorar las condiciones de vida o de salud y, por ende, no son de fácil renuncia o eliminación de la cesta de productos que se van a adquirir. El tema radica en evidenciar si efectivamente se logra dicho goce: "En el campo internacional, ha tenido lugar un incremento en la aplicación de las evaluaciones farmacoeconómicas para cuantificar el valor de los servicios y productos farmacéuticos en el contexto de los sistemas nacionales de salud" (2).

La conducta del consumidor de medicamentos debe ser mediada por las curvas de indiferencia o satisfacción, como se denominan en la economía, sobre las cuales el consumidor siempre estará dispuesto a mejorar sus condiciones iniciales para alcanzar mejor bienestar. Si este escenario no llega a cumplirse de manera plena, es claro que el consumidor se encuentra sujeto a las prerrogativas empresariales y del mercado. "En todo el mundo, crecen las evidencias del impacto de esos problemas sobre la población y sobre los sistemas de salud" (3).

La salud es de los pocos negocios en los que la rentabilidad es evidente, y de paso a ello, la solicitud de mejores y nuevos productos es una constante. Por ende, es de considerar que la participación en este tipo de empresas obedece más a la expectativa de ganancia que a la motivación social o de salud que en principio podría ser su objeto central. Tras de estas compañias se encuentran diferentes inversionistas, cuya meta se centra en la ganancia por el capital invertido. "Se conoce que la incorporación del modelo de desarrollo capitalista y neoliberal a la industria farmacéutica ha cambiado la forma en la que hace llegar los medicamentos que produce a la población" (4).

La generación de medicamentos para todo tipo de enfermedades, tratamiento o para la salud obedece no solo a condiciones de oferta y demanda, como igualmente sucede por el lado del consumidor, sino que la ciencia, la tecnología y la innovación hacen parte de los sustratos que evidencian el recorrido que debe hacer un medicamento para disponerse en el mercado, previa la barrera de entrada de las patentes. "De esta manera, el descubrimiento y el desarrollo de nuevos medicamentos por la IF están obligados a servir no solo a intereses científicos o médicos, sino también a la eficiencia económica" (5). 
El contenido económico del sector empresarial e industrial químicofarmacéutico, en cuanto a la generación, obtención y producción para de medicamentos para la salud humana y animal, no es fácilmente evidenciable desde la condición económica básica de demanda, oferta y precios. Se trata de una economía cuyo bien central o satisfactor es la salud, que como bien hedónico o de plena satisfacción, nadie está dispuesto a mermar o a renunciar. "Drugs that had larger increases in international price dispersion had larger increases in total utilization, controlling for the growth in the mean price of the drug and the drug's vintage" (6).

La errática situación de los sistemas de salud en el mundo y la observancia sobre cada uno de los sectores de la cadena ha puesto entre otras, a ellas, en el centro de las controversias, por estar más ubicadas en el negocio que en la salud. La metodología de la selección adversa, el riesgo moral y la teoría de la agencia orienta el propósito general de la investigación. "El comportamiento del mercado fármaco-terapéuticos ha sido objeto de estudios por distintos economistas, para realizar explicaciones académicas sobre el comportamiento de los monopolios en el mercado" (7).

La política de medicamentos, la estructura empresarial, las evaluaciones hechas a la tipología de suministros y la clase de elementos, en cuanto resultados efectivos y eficaces que corren por cuenta de las empresas químico-farmacéuticas, no son visibles a la luz de la eficacia y los cometidos que se espera respecto a la disposición de medicamentos para el bienestar y la economía de la salud. "Debe mencionarse que esta dinámica del gasto en salud no es exclusiva de Colombia, pues ya hace varias décadas países como Inglaterra, con un fuerte peso de recursos públicos en la salud" (8).

El usuario final no percibe con claridad la efectividad de los medicamentos que le son proveídos o suministrados por agentes intermediarios o productores de medicamentos a escala internacional. Prevalecen fisuras que no permiten que haya concordancia entre los fines de los medicamentos con los resultados esperados sobre ellos, y que hoy extienden un manto de duda sobre las empresas químico-farmacéuticas. "En salud, el Análisis de Impacto Presupuestario (AIP) busca calcular los resultados financieros de adoptar y difundir una intervención en un programa de cobertura específica o sistema de salud" (9).

La posibilidad de interiorizar el accionar de estas con respecto a la economía, la geopolítica y los propios sistemas de salud encaran el propósito de esta investigación y la posibilidad de que, con cargo a ello, se plantee un análisis amplio en el país. Al identificar los criterios, los fundamentos y los preceptos que guían a las compañias, empresas, firmas y marcas del sector químico, "el modelo que se presenta para el análisis de la industria de los productos farmacéuticos acumula la investigación histórica al respecto y deja abierta la posibilidad de una contrastación empírica posterior” (10).

La estructura empresarial e industrial que compone al sector farmacéutico y químico preserva de manera transversal la correlación entre oferta de productos para la salud con respecto a la economía de la salud, razón por lo que se hace necesario conocer el alcance, el contenido y el propósito de las compañias que estas al frente de dicho rango del mercado. No obstante, 
el escenario contemplado pretende identificar los bastiones sobre los cuales se yerguen en los sistemas de salud en el mundo. "La farmacoeconomía es la aplicación del análisis económico en el campo de los medicamentos” (11).

El contenido analítico expuesto por las teorías de la selección adversa, el riesgo moral y la teoría de la agencia-principal sintetiza la naturaleza del comportamiento de las estructuras empresariales confinadas a la actividad e industria química-farmacéutica. Así, al evaluar dichos elementos conforme al desempeño individual, colectivo y social de las estructuras mencionadas puede llegar a establecerse claramente que las empresas ubicadas allí, independiente a su actividad, deben procurar mediar con externalidades. "E1 acceso universal a los medicamentos no es una operación económica, aunque debería ser económicamente viable" (12).

La selección adversa permite identificar los parámetros que toman las empresas y firmas de la industria para apostar por unos medicamentos que cumplan su cometido entre unos mínimos o máximos. El riesgo moral aparece cuando, ajenos a sus cometidos, se entremezclan otras intenciones, escenario en el que se hace a un lado su cometido, y comienza el interés por ubicar su quehacer en términos de la ganancia. Asimismo, la teoría de la agencia-principal identifica la captura a la que ha sido sometido. "La solución de largo plazo para la situación farmacológica es el desarrollo de una forma de organización social que efectivice el control sobre los recursos mundiales" (13).

De ahí que se entienda que al intentar establecer una relación directa con estas firmas al amparo de sus intereses o revisión de estos, sea necesario llegar a puntos de equilibrio en que el interés individual que ellas estiman no afecte la disposición de medicamentos y, de paso, que con cargo a ellos su impacto y utilidad cumplan su cometido inicial para el caso colombiano o de otro país. "Como aspectos relevantes se destaca el esfuerzo por crear un sistema de salud con un paquete de medicamentos adscritos a los que los colombianos tendrían acceso en términos de disponibilidad y precios" (14).

Es de aclarar que se abre el espacio para revisar diferentes formas empresariales inmersas en cualquiera de los estertores que componen la industria química-farmacéutica. Frente a ello, la labor consiste en postular mecanismos por medio de los cuales pueda abrigarse a las empresas como cadena del sector salud en general. En términos generales, la salud no se ve integrada, a pesar de que acuña el concepto de sistema de salud, dado que cada uno de sus agentes toma una referencia del sistema para plantear posturas frente a este, y en los que claro está la industria química-farmacéutica también tiene un espacio propio, pero en el sistema como es de esperarse. "Muchos de los progresos requeridos en los campos mencionados se asocian a acciones en el plano universitario, en el de las cátedras hospitalarias, y en el de los laboratorios farmacológicos del mismo sector público" (15).

En cuanto a la revisión de las empresas conforme a su desempeño en el sistema de salud colombiano, la idea es poder integrar diferentes conocimientos y fundamentos tras los cuales pueda explicarse, facultarse y provocar ejercicios en los cuales la gestión las firmas químico-farmacéuticas pueda ser, a la par de estudiada, revisada, en aras de su fortalecimiento. 
"La industria farmacéutica colombiana, la cual agrupa a los subsectores de medicamentos y fitoterapéuticos $(1,2)$, entre otros aspectos se caracteriza por su gran dependencia tecnológica" (16).

La revisión del componente económico de los medicamentos en el sistema de salud colombiano ubica necesariamente la investigación en el análisis del concepto de farmacoeconomía, y cómo este, conforme a los preceptos sobre los cuales se pretende fundamentar, puede contribuir a depurar las dificultades que se tienen en cuanto a la poca sinergia entre la oferta de medicamentos y los sistemas de salud. "A juzgar por la forma como se ha desarrollado la farmacoeconomía, prevalece el enfoque de la rentabilidad económica" (17).

El seguimiento hecho a los impactos de los medicamentos corresponde habitualmente a evaluaciones de autoridades en países desarrollados o a autoevaluaciones que sobre las mismas prescripciones realizan de manera seria algunos laboratorios, y pese a los esfuerzos por interiorizar el papel del consumidor en las estrategias y politicas con respecto al conocimiento e información sobre los medicamentos, la reciprocidad es nula.

\section{Conducta y estructura del consumidor de medicamentos y productos farmacéuticos}

En este caso, el interés, bajo información completa, es considerar el consumo de productos farmacéuticos, de laboratorios y medicamentos hallados en la canasta de bienes, sin ningún tipo de dificultad para acceder a ellos. Este maximiza su utilidad con respecto a la combinación de dichos bienes en canastas o cestas de acuerdo con los precios conocidos y restricción presupuestaria, que, en últimas, tratan de estructurar el campo económico del sector. "La farmacoeconomía es la determinación de la eficiencia (relación entre costo y efectos) de un tratamiento farmacológico y su comparación con las de otras opciones" (18).

La argumentación planteada sobre el consumidor racional indica que este conoce la renta, la oferta de productos y los precios de los bienes requeridos para maximizar su bienestar; pero, en sí, desconoce la cantidad de productos que se van a adquirir. Por ende, el consumidor espera que la adquisición de medicamentos, igualmente, maximice la utilidad esperada de acceder e ingerir los medicamentos que requiere. "Además, este no se preocupa demasiado por su coste y el medicamento tampoco es pagado en gran parte por el paciente, sino por el Gobierno" (19).

En este marco, los bienes representan una canasta compuesta, igualmente representativa, aglutinada por otra serie de bienes que identifican la canasta principal. La educación, la salud y la vivienda representan cestas de bienes compuestos en las que se encuentran inmersos otros bienes adheridos, en los que debe tomarse en cuenta el agregado de la salud. "El acceso a los medicamentos tiene también un alto impacto sobre los servicios, ya que son un insumo básico del proceso terapéutico" (20).

En el entendido, si la salud hace parte de la canasta de bienes compuestos por parte del consumidor, de manera efectiva deben conocerse los resultados generados por dichas canastas - en este caso de los medicamentos- sujetos a la naturaleza de los bienes ubicados en las canastas. "Las 
características del consumo de medicamentos son bien definidas por estrato socioeconómico" (21).

El concepto de maximización de utilidad o satisfacción está consagrado para informar respecto a las relaciones cardinales y ordinales en las que el consumidor, a través de su libre elección o preferencia, decide adquirir una u otra cesta prevista en el mercado de los medicamentos de salud, que para este caso están coligados a otros factores. "Estos elementos son de diferente indole (jurídica, institucional, política, administrativa, informativa, etc.) y constituyen el denominado marco institucional del mercado" (22).

En este frente, la salud referida a los medicamentos presenta amplios atrasos explicativos en la materia. En ella hace una evaluación plena de las posibilidades brindadas por las distintas combinaciones de canastas o productos inmersos en ellas, aspecto sobre el cual toma la decisión de elegir una de dichas interacciones para lograr la maximización de la utilidad o satisfacción conforme al bien adquirido. "Los medicamentos son bienes de consumo. Pero no son normales. Desde un punto de vista económico registran particularidades que los diferencian de cualquier otro bien" (23).

La plena información apunta a la inexistencia de costos de transacción o externalidades que afecten las decisiones tomadas por el consumidor a la hora de preferir sus canastas de consumo. En primer lugar, la exposición a una relación contractual o de contratos para acceder a la cesta de productos farmacéuticos y médicos expone al consumidor a no tomar eficazmente decisiones plenas en la materia. Segundo, la aparición de externalidades puede generar alteridades de la información que implican un esfuerzo mayor para el consumidor a la hora de reconocer los medicamentos idóneos. "Las evidencias científicas permitirán fundamentar una selección racional y evitar confrontaciones con el sistema judicial y con los pacientes, y habilitarán al Estado o a las aseguradoras no solo a pagar por productos que realmente tengan suficientes efectos terapéuticos positivos" (24).

En este punto, la característica alude a una intrincada red de interacciones que, antes de obtener productos, implica que el costo o los precios para acceder a ellos supera las relaciones básicas previstas por las condiciones básicas de mercado relacionadas entre la oferta y demanda de los productos sujetos al precio, en las que el producto es superado por las transacciones de no mercado de los costos. "Para control de precios de medicamentos es necesario contemplar a la cadena de producción y comercialización" (25).

Por cuenta de las externalidades se advierten hechos ajenos a los consecuentes con las condiciones y posibilidades de lograr la adquisición de las canastas, en las que los diversos consumidores puedan ser atendidos eficazmente. Dichas externalidades exponen al producto a tener dificultades de intercambio en el propio mercado y que, de paso sea dicho, impiden su consecución o producción e incluso su neutralidad. "La crisis económica y la necesidad de contención del gasto sanitario exigen reducir drásticamente la tendencia a originar fuertes incrementos en la factura farmacéutica financiada públicamente" (26).

La canasta de la salud en materia de productos farmacéuticos y medicamentos no dispone de vasta información tras de la que, de manera objetiva, 
puedan abrigarse las distintas combinaciones que, para el efecto, decanta la microeconomía a la hora de abordar un sector tan especial como este, en el que se requiere el equilibrio de todos los demás. "Es evidente: el medicamento constituye un importante determinante de la salud de los ciudadanos, aunque solo fuera porque de ellos depende en buena medida la capacidad que hoy tiene la Medicina de interrumpir o modificar el curso natural de las enfermedades" (27).

En este frente, es imperante que el análisis vinculado a este sea guiado por la disposición académica de la microeconomía que, conforme a su interés por orientar al consumidor hacia diferentes mecanismos de maximización del consumo, pueda tener diferentes opciones para hacer frente a sus requerimientos en el tema. Precisamente, es un campo en el que se requiere una evaluación mucho más diversificada que la sola atomización en la canasta. "La farmacoeconomía es muy valiosa para la realización del consumo farmacéutico hospitalario" (28).

A efectos del análisis general, es prudente asumir que la canasta de productos farmacéuticos y medicamentos, igualmente, responden a la cesta conferida para hacer alusión tanto a la salud animal como a la humana, conforme a la teoría del análisis racional del consumidor, en la que se destacan la satisfacción o la utilidad, no los precios. "We performed an empirical investigation of whether the necessary condition for price discrimination to increase welfare-that it increase total output-is satisfied in the case of international pharmaceutical prices" (6).

El consumidor es exógeno a las interpretaciones que, en materia económica, productiva o social, realizan las empresas involucradas en el sector farmacéutico. Precisamente, dicha exogeneidad implica la imposibilidad de generar condiciones de equilibrio del consumidor o de oferta y demanda para estos casos. El consumidor tiene dificultades para reconocer la información disponible en materia de productos farmacéuticos y de medicamentos.

\section{Conducta y estructura empresarial de los productores farmacéuticos y de laboratorios químico-farmacéuticos}

La conducta empresarial en la microeconomía explica el comportamiento de los productores farmacéuticos y los laboratorios, en cuanto a la optimización de la producción y minimización de costos, bajo la búsqueda permanente de maximización de beneficios económicos, tríada que define la estructura empresarial a la que responden (figura 1).

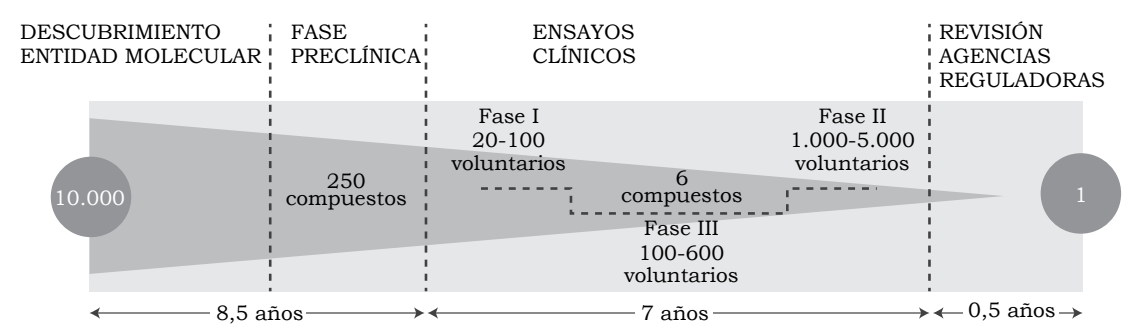


Es fundado el papel que cumple la combinación de capital y trabajo en el sector farmacéutico y de laboratorios químicos en la provisión de productos para la salud animal y la humana o el complemento de estos en otros menesteres. Ambos, representan elevados desembolsos en recursos, tanto en equipos como en generación de conocimiento, además del tiempo que se requiere para la obtención de licencias, marcas, patentes y registros. "La Organización Mundial de la Salud (OMS) define a los fármacos, medicinas, productos farmacéuticos y productos medicinales como aquellas sustancias o productos utilizados en el ser humano con fines profilácticos, diagnósticos o terapéuticos" (30).

La consecución de los factores de producción, a la par con las materias primas, reviste para las empresas del sector la prioridad de desembolsar recursos que, a diferencia de lo que sucede en la producción fabril o de transformación industrial, no implica necesariamente la obtención de un resultado concreto, como es de esperarse en el sistema de salud. "De hecho el desarrollo de investigaciones en el campo de la farmacia asistencial utilizando la metodología cualitativa en combinación con las ya aplicadas técnicas cuantitativas" $(31,32)$.

La maximización del producto en la actividad de la empresa farmacéutica y de laboratorios contempla el comportamiento reconocido por la productividad marginal, media y total, característica de la microeconomía de la empresa en la que se destaca la ganancia, el ingreso marginal y el beneficio. "Obviamente, esto conlleva dos modelos polares de organización, conformados, el primero de ello por mega empresas con una alta integración técnica y productiva (especialmente en las actividades nodales) y, el segundo, por la segmentación de actividades en el marco de tramados productivos más desconcentrados" (19).

En toda su dimensión, los costos ofrecen información pertinente para explicar la fundamentación que estos crean en sectores en los que la consecución de beneficios tarda por razones institucionales, que desbordan el interés del mercado en cualquier tipo de producto o sector como el de los medicamentos. "El costo fijo de desarrollo de un nuevo medicamento es extremadamente alto por dos razones: es muy caro investigar y afinar un medicamento nuevo y luego hacerlo pasar por pruebas clínicas complejas y procesos nacionales de aprobación" (33).

Gran parte de la respuesta obtenida en dicho propósito se alcanza en la medida en que se obtienen resultados concretos, en cuanto a las investigaciones trazadas o aplicativos que en el laboratorio o resultados de ensayos, tras de los que efectivamente se obtengan pruebas alentadoras, que no necesariamente implica reducción de costos. "La evaluación económica de medicamentos constituye un instrumento que se utiliza cada vez con más fuerza en la toma de decisiones" (34).

La combinación entre la maximización del producto total y el costo total permite analizar las condiciones en que se encuentra la empresa frente a la posibilidad de continuar produciendo de manera progresiva o definir criterios de producción que no impliquen perder la optimización de la producción o la minimización de los costos. "¿Cuál es el poder del mercado, 
del paciente, de los colegas y asociaciones científicas sobre las decisiones de prescripción?" (35).

La revisión de la estructura de producción de este tipo de empresas es crucialmente relevante, si se comprende el comportamiento de estas. Así como lo comentado con respecto a la conducta de los demás elementos, las empresas ubicadas en el margen del sector de los productos químico-farmacéuticos ofrecen criterios diferentes a la cotidianidad enmarcada en la teoría del productor. Por ende, el análisis implicado en este campo debe tener un marco de elaboración superior al conocido por el momento de manera habitual. "Las farmacéuticas han olvidado que existen regiones y países que carecen de medicamentos para atacar o contrarrestar los padecimientos de sus pobladores" (36).

Es crucial para el mercado definir los claros márgenes de ganancia del sector y el beneficio económico derivado de la interacción entre ingresos totales y costos totales, pues ello determina el resultado del ejercicio productivo de manera económica. "El desempeño productivo y comercial farmacéutico de Argentina, Brasil y México es diferenciado, pero a los tres los caracteriza la ausencia de una indus $\neg$ tria integrada con un sector farmoquímico capaz de generar los insumos que la industria requiere (tal vez México en mayor grado)" (37).

La provisión de medicamentos está sujeta a la capacidad industrial, en cuanto a capital y trabajo, que dispongan las empresas establecidas en este rango, y que de paso, deben ser de alto nivel y conocimiento. En ellas, el comportamiento de la relación productiva con los costos infiere para permitir la producción y, de paso, corroborar la generación de excedentes a través de la relación diferencial entre ingresos y costos (figura 2).

LA ESCALERA TECNOLÓGICA

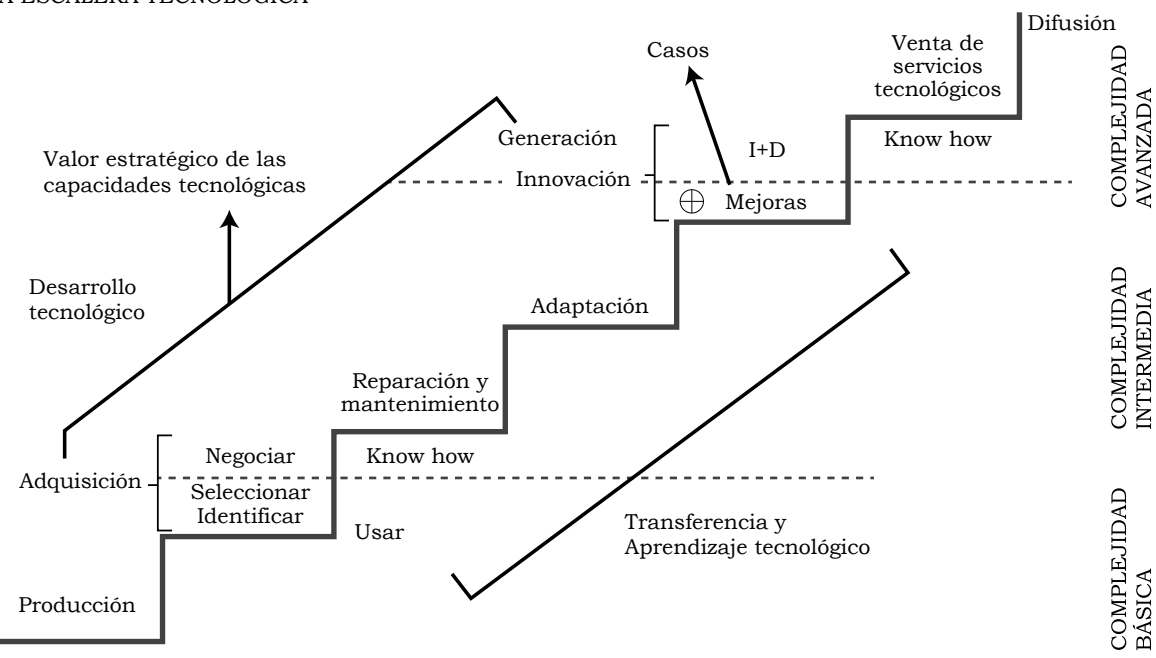

Figura 2. Escalera tecnológica

164 Fuente: Vargas y otros (2003), citado en Vallejo y Torres (38). 
Las compañias ubicadas en este rango se especializan en áreas de trabajo específico en las que terminan expertas; de paso, su actividad les permite avanzar en diferentes escalas de su producción en las que generarían excedentes, y de paso, además de solventar su propia actividad, pasarían a jalonar a las compañías ubicadas en sus rubros de producción:

El precio está intervenido en casi todos los países de la UE a excepción de Dinamarca, Alemania y Suecia, donde hay libertad de precios. E1 coste de los medicamentos consta de varios componentes: el precio de venta del laboratorio, el margen del mayorista, el margen de la oficina de farmacia y el IVA. (22)

La adquisición de dichas referencias se logra mediante la compra de productos de marcas o genéricos. La disputa entre dichas empresas por productos y marcas evade los requerimientos en materia de salud; de paso, el comportamiento del mercado no es compatible con las expectativas que en materia de salud deberia arrojar el sector. "Our findings show that as market competition intensifies, entering start-ups will be more likely to file initial IPs in the form of a trademark and less likely to file an initial IP [intellectual property] in the form of a patent" (39).

En su defecto, corresponde por la vía del marco jurídico o la regulación exponer los principales elementos que deben acompañar su actuación y quehacer, y no solo arrinconarlas al escarnio generalizado que da cuenta de su interés por los excedentes y ganancias de la producción de medicamentos. El asunto aquí se resuelve a través del análisis contractual, de riesgo moral, selección adversa y desde el análisis de la teoría de la información.

\section{Definición de la estructura del equilibrio sectorial del mercado farmacéutico (consumidores y empresas)}

Circunstancias puntuales y casos excepcionales han puesto la imagen de estas empresas en la mira de las autoridades institucionales y organismos multilaterales, como la Organización Mundial de la Salud y el Banco Mundial, que han sugerido a los países revisar la composición de los productos que por cuenta de las empresas químico-farmacéuticas son ofrecidos en los dispensarios y el impacto en la salud. "La atención farmacéutica es la provisión responsable de la terapia medicamentosa con el propósito de alcanzar resultados definidos que mejoren la calidad de vida del paciente" (40).

Si bien su disposición, oferta y prescripción han aumentado ostensiblemente, no ha tenido igual correspondencia en el mejoramiento de los estándares en salud o que, por lo menos, evidencien qué tanta efectividad han tenido en la salud de los pacientes en los países en los cuales tienen prevalencia como oferta directa por toda la cadena de valor en salud. "Se deberian potenciar los centros alternativos de información y actualización en farmacoterapéutica, independientes de la industria, que provean información a los prescriptores, a los consumidores y a los que dispensan los medicamentos" (41). 
Es evidente la estructura oligopólica desde la cual se extiende la presencia de diferentes empresas, laboratorios, marcas y productos cuya procedencia destaca a las empresas e industria químico-farmacéutica como el eslabón central de la cadena; por lo tanto, el diálogo, la evaluación o la regulación en este frente debe ser de manera directa o concertada. "El nuevo contexto económico y social internacional tendrá probablemente un efecto importante sobre el acceso equitativo de las poblaciones a la salud y a los medicamentos, particularmente en los países en desarrollo" (42).

De la revisión que se haga al respecto, depende no solo su continuidad en el tiempo, sino la posibilidad de interiorizar aún más su capacidad de acción. De lo contrario, valdrá la pena de nuevo de facultar o aprovisionarse de medicamentos por cuenta de casas o empresas que garanticen efectivamente los impactos y resultados prescritos en sus productos (figura 3).

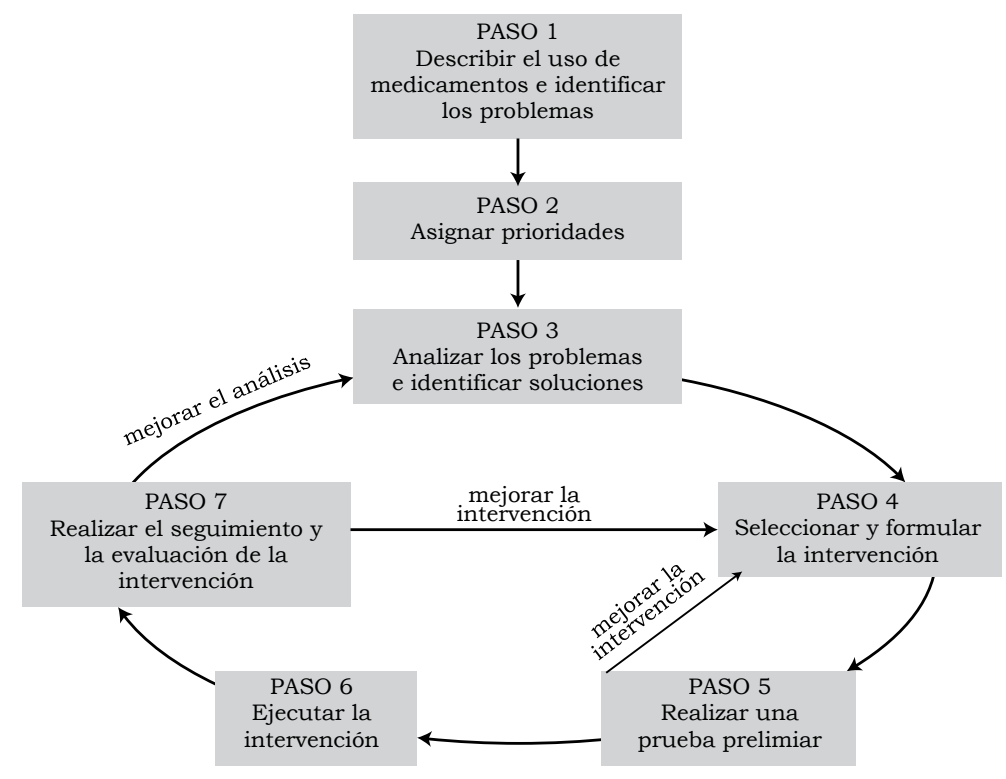

Figura 3. Pasos en la formulación de una intervención eficaz dirigida a promover el uso racional de los medicamentos entre los consumidores

Fuente: Hardon, Hodgkin y Fresle (43).

La idea es ubicar la discusión en un punto desde el cual puedan extenderse las características de un sector que -alusivo a las propias temáticas a las que responde - se mueve de manera oculta y de espalda a la realidad de la salud de los pacientes y de los propios sistemas. Parte de la discusión pasa no solo por la disposición de los productos de las grandes marcas, sino por la efectividad en reparo de estos (figura 4). 


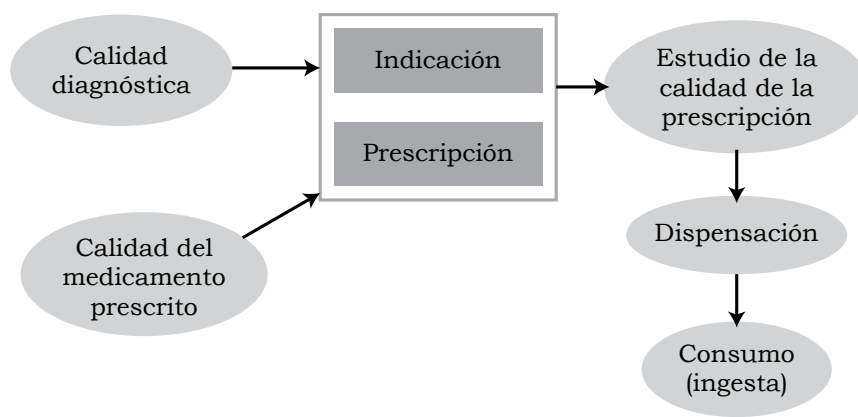

Figura 4. fases de la utilización de los medicamentos

Fuente: tomado de López et al. (35).

El cuestionamiento acerca de los derechos de propiedad intelectual y las patentes ha sido parte de la discusión que ha concentrado el interés de los laboratorios de las grandes marcas internacionales y las empresas de genéricos en el mundo. Los primeros abogan por el planteamiento de exigencias y requisitos mucho más amplios para las empresas de genéricos, y los segundos indican que su vigencia en el mercado tiene su explicación en los altos costos (figura 5).

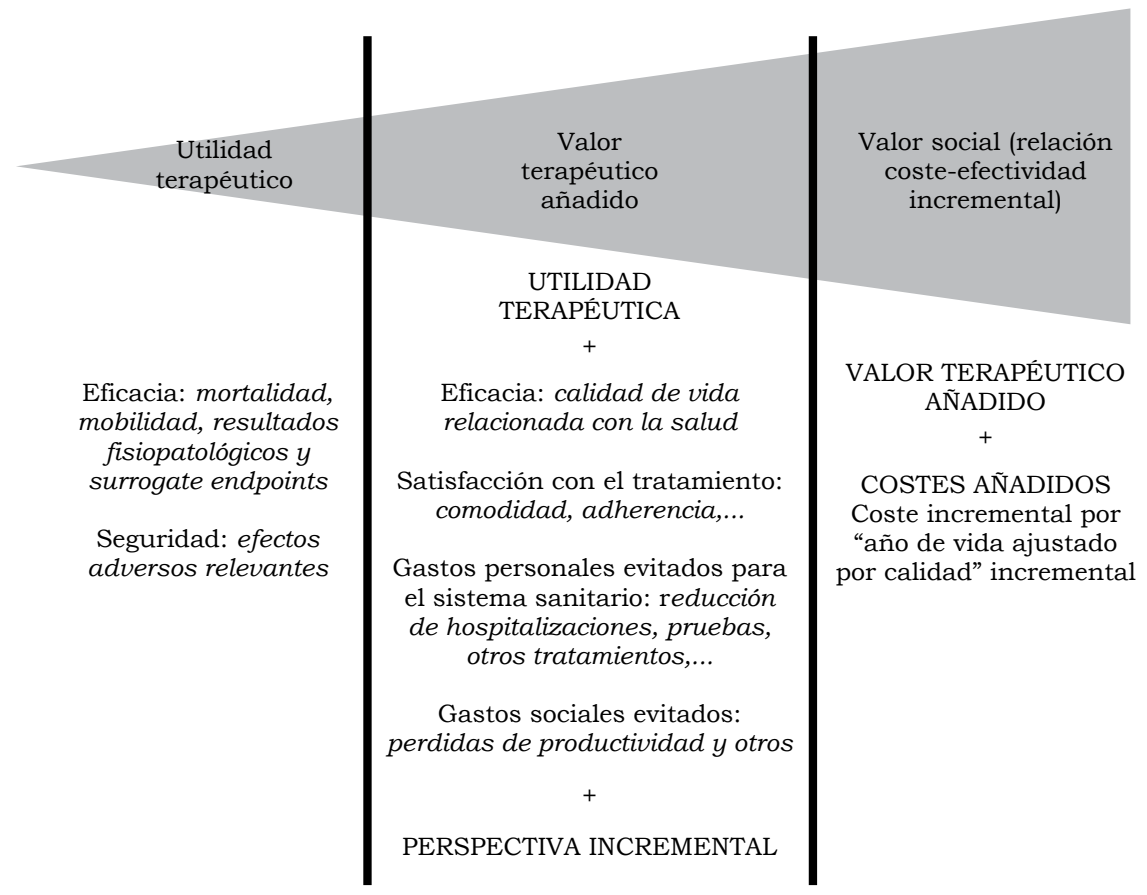

Figura 5. Utilidad terapéutica, valor terapéutico añadido y valor social de los medicamentos Fuente: Puig y Peiró (44) 
Al respecto, prevalecen serios interrogantes que tienen a estas empresas en un proceso profundo de revisión que debe tener respuesta en ellas mismas, más que desde otros frentes, que si bien señalan, poco impactan mientras ellas no se lo replanteen, aparte de si realmente dichas empresas independientes a su estructura están al tanto de las bondades de ofrecer medicamentos efectivamente para la salud. "El objetivo final de todo el proceso de gestión de los riesgos asociados al uso de los medicamentos es minimizarlos" (45).

Las empresas de la industria químico-farmacéutica están pasando por un escrutinio mundial con respecto a los resultados obtenidos por cuenta de las medicinas producidas por ellos, pero en muchos casos el resultado obtenido termina siendo contraproducentes a las perspectivas creadas en esencia. "Para el resto del sector productivo farmacéutico, incluidas las pequeñas y medianas empresas, pero también para una parte de las grandes empresas, esa prioridad es muy discutible y quizá debería completarse el diseño de la política industrial para el grupo selecto" (22).

Las firmas se han ubicado al extremo "débil" de la cadena, al disponer su actividad desde la enfermedad y no en procura de la salud, epílogo que ha servido para que en la actualidad se cuestionen los resultados obtenidos por las diferentes empresas del sector, y que han llevado a una revisión de parte de los ministerios de salud para contrarrestar dicha situación. "Con abundancia de datos cuantitativos, el autor ratifica la posición monopólica de la industria farmacéutica internacional" (46).

Si la mayoría de medicamentos no contiene los aditamentos necesarios para curar, resarcir o sanar dolencias o la mitigación del dolor en casos tanto generales como particulares, no solo está en riesgo la credibilidad de las empresas dedicadas abiertamente a este negocio, sino que en gran medida están comprometidos los sistemas de salud propios de los países que dan vía libre a la comercialización de productos considerados idóneos y necesarios. "En el caso de los medicamentos, la mayoria de las poblaciones de los países menos desarrollados sin cobertura estatal o de sistemas privados de seguros médicos tienen que afrontar con sus bolsillos la compra de medicamentos" (47).

El propio escenario de acceso, disposición y suministro de medicamentos está siendo puesto en duda, al presentarse diversos casos vinculados con la animadversión a múltiples medicamentos o la aparición de enfermedades por cuenta de la posología o suministro por quienes de modo manifiesto hacen parte de la cadena de valor de la industria. "La economía de la salud analiza los aspectos económicos de la atención sanitaria mediante métodos y teorías económicas y de ciencias de la salud" (48).

Si en algún momento la industria química-farmacéutica se ha catalogad como el dispensario en materia medicamentos vinculados con la provisión de medicinas, el paso de la disposición al mercado ha terminado por ubicarla en el papel de factor de incidencia en los resultados obtenidos en materia de salud (figura 6). 


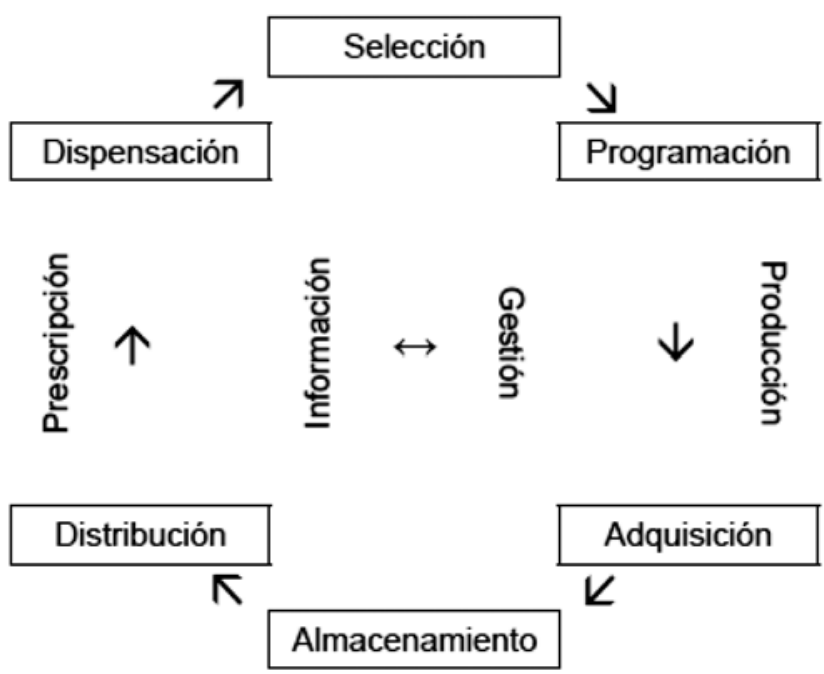

Figura 6. Ciclo de la asistencia farmacéutica

Fuente: tomado de Tolentino (49).

No obstante, entre la cantidad de ellas y el conocimiento que se tiene sobre el impacto efectivo que arrojan los medicamentos, ha empezado a hacer carrera la discusión sobre el impacto de estos en la salud y qué tanto cumplen realmente su cometido conforme a la prescripción que asiste en ellos. "En Atención Farmacéutica la misión del farmacéutico asistencial, se desplaza desde el proceso de dispensar medicamentos al de la atención al paciente" (50). El desconocimiento sobre estos temas hace carrera en un mundo, en el cual la atención en la disposición del suministro de los medicamentos atraviesa serios cuestionamientos respecto a su eficacia y contundencia, en cuanto al bienestar en salud.

\section{Conclusiones}

El consumidor de medicamentos y productos de la gran industria farmacéutica y de laboratorios desconoce por completo la movilidad que en materia de beneficios trae el ubicarse en este renglón industrial. En dicho sentido, el consumidor contempla dicho mercado como bienes hedónicos o meritorios. El interés de las multinacionales y transnacionales resulta estrictamente económico y financiero, debido al apalancamiento financiero y accionario explícito en estas compañias, que finalmente son medidas por cuenta de los resultados económicos y financieros, por encima de los propósitos sociales o vinculados a la salud. A eso se suman los problemas de agencia, caracterizados por las múltiples organizaciones que representan a unos y otros, hecho que expresa el evidente problema de institucionalidad efectividad del sector, en la que en ninguno de los dos se logra un grado de equilibrio o satisfacción por la labor lograda en ambos flancos centrales de la cadena de medicamentos. 


\section{Financiación}

Proyecto financiado por la Dirección de Gestión del Conocimiento de la Universidad CES en Medellín (código INV032013003), entre el 2 de julio de 2013 y el 2 de julio de 2014, ejecutado por el grupo de investigación en Gestión Empresarial, reconocido categoría D en Colciencias; el Grupo de Perdurabilidad Empresarial de la Universidad del Rosario, reconocido en categoría A, y el Grupo Investigación en Ciencias Farmacéuticas-CES (ICIFCES), de la Facultad de Química Farmacéutica de la Universidad CES.

\section{Conflicto de interés}

Ninguno

\section{Referencias}

1. Román V, Di Salvo MT. La producción de medicamentos en Argentina: notas para su análisis. SaberEs. 2010;(2):73-93.

2. Collazo HM, Sosa LI. La farmacoeconomía: ¿debe ser de interés para evaluar la eficiencia en la toma de decisiones? Rev Colomb Cienc Quím Farm. 2011;40(1):54-66.

3. Januario CC, Soler O, Fleith OM. Servicios farmacéuticos integrados al proceso de cuidado en salud: gestión clínica del medicamento. Rev Pan-Amaz Saude. 2011;2(3):41-49.

4. Rodríguez A. La realidad detrás de las farmacéuticas: sendas de una posmodernidad que no perdona. Rev Enferm Actual de la Costa Rica [internet]. 2015;(29). Disponible en: http://www.revenf.ucr.ac.cr/farmaceutica.pdf.

5. Páez R. La investigación de la industria farmacéutica: ¿condicionada por los intereses del mercado. Acta Bioethica. 2011;17(2):237-46.

6. Lichtenber FR. Capitalism and society: pharmaceutical price discrimination and social welfare. Columbia University and National Bureau of Economic Research. 2010;5(1):1-29.

7. Stepanian SM, Tirado RA. Oportunidades y amenazas para el sector farmacéutico con la firma del TLC con los Estados Unidos. Bogotá: Universidad EAN Facultad de Ciencias Económicas; 2013.

8. Castañeda C, Fonseca M, Núñez J, Ramírez J, Zapata JG. La sostenibilidad financiera del sistema de salud colombiano: dinámica del gasto y principales retos de cara al futuro. Bogotá: Fedesarrollo; 2012.

9. Garay OU, Caporale JE, Pinchón RA, García MS, MacMullen M, Augustovski F. El análisis de impacto presupuestal en salud: puesta al día con un modelo de abordaje genérico. Rev Peru Med Exp Salud Pública. 2011;28(3):540-7.

10. García RM. Un modelo de análisis competitivo del sector farmacéutico. Investigaciones Europeas de Dirección y Economía de la Empresa. 1995;1(3):103-11.

11. Gálvez GAM. La farmacoeconomía en la eficiencia de la salud pública. Rev Cubana Salud Pública. 1999;25(1):81-85.

12. Lage A. Desarrollo farmacéutico global y acceso a medicamentos: temas críticos de ética y equidad. Medicc Review [internet]. 
2011;13(3):1-8. Disponible en: http://www.medicc.org/mediccreview/ index.php?issue $=17 \& i d=204 \& a=$ va .

13. Bodenheimer TS. La industria farmacéutica internacional y la salud de la población mundial. Cuadernos Médico Sociales. 1983;(24):1-13.

14. Vásquez VJ, Gómez PK, Rodríguez AS. Regulación en el mercado farmacéutico colombiano. Revista de Ciencias Sociales (RCS). 2010;16(2):197-209.

15. Katz JM. Estadios de desarrollo e industria químico-farmacéutica. Cuadernos Médico Sociales. 1981(18):1-17.

16. Vallejo DBM, Torres PNS, Rivera PJE, Carvajal MF, Bolivar DC. Estudio descriptivo de los subsectores productores y comercializadores de medicamentos y fitoterapéuticos en Bogotá. Rev Colomb Cienc Quím Farm. 2007;36(2):175-91.

17. Velásquez G. Farmacoeconomía: ¿evaluación científica o estrategia comercial? Rev Panam Salud Publica.1999;5(1):54-7.

18. Collazo HM, Flores DN. Farmacoeconomía, evaluación de la eficiencia en los tratamientos farmacológicos. Rev Cubana Farm. 2000;34(1):63-9.

19. Barrutia X, Zabalo P. Sector farmacéutico, patentes y acceso a medicamentos en el sur. Revista CIDOB d'Afers Internacionales. 2003;(64):1-15.

20. López MS, Martínez Ojeda RH, López AO, Jarillo SE, Castro AJM. Organización del abasto de medicamentos en los servicios estatales de salud: potenciales consecuencias de la mezcla público-privada. Salud Pública Mex. 2011;53(4):445-457.

21. Mestanza F, Pamo O. Estudio muestral del consumo de medicamentos y automedicación en Lima metropolitana. Lima: Departamento de Medicina, Universidad Peruana Cayetano Heredia; 1990. p. 1-10.

22. Antoñanzas F, Rodríguez R, Sacristán JA, IIla R. Los medicamentos en la Unión Europea: el tándem comercio-salud. Gac Sanit. 2005;19(2):151-67.

23. Tobar F, Sánchez D. El impacto de las políticas de medicamentos genéricos sobre el mercado de medicamentos en tres países del Mercosur. Montevideo: Centro de Informaciones y Estudios del Uruguay (CIESU); 2007. p. 1-73.

24. Marín GH, Polach MA. Medicamentos de alto costo: análisis y propuestas para los países del Mercosur. Rev Panam Salud Pública. $2011 ; 30(2): 167-76$.

25. Tobar F. Asistencia técnica a las autoridades nacionales presentando propuesta de metodología para fijación de precios de medicamentos al sector farmacéutico [informe final misión 23] [internet]; 2011. Disponible en: https://www.google.com.co/url?sa=t\&rct=j\&q=\&esrc= s\&source $=$ web\&cd $=1 \&$ cad $=$ rja\&uact $=8 \& v e d=0 \mathrm{CBwQFjAAahUKEwjeq}$ MLZwPLHAhWBJR4KHbPNCUQ\&url=http\%3A\%2F\%2Fwww.mincit. gov.co\%2Fdescargar.php\%3Fid\%3D62393\&usg=AFQjCNHj5vrHMBA jq97kuL8SZkz_KdS59A\&bvm=bv.102537793,d.dmo

26. Iñesta A, Oteo LA. La industria farmacéutica y la sostenibilidad de los sistemas de salud en países desarrollados y América Latina. Ciência \& Saúde Coletiva. 2011;16(6):2713-24. 
27. Abajo F . El medicamento como solución y como problema para la salud pública: una breve incursión a los objetivos de la farmacoepidemiologia [editorial]. Rev Esp Salud Pública. 2001;75(4):281-4.

28. Lichtenberg FR. Pharmaceutical price discrimination and social welfare. Capitalism and Society. 2010;5(1).

29. Cuello de Oro DJ, López-Cozar NC. I+D y rentabilidad empresarial: una relación circular en el sector farmacéutico. Revista Europea de Dirección y Economía de la Empresa. 2011;20(2):23-34.

30. Ramírez RMO. La prescripción de medicamentos y su repercusión social. Rev Cuban Salud Pública. 2006;32(4):1-12.

31. Ramalho de Oliveira D, Dupotey VN. La investigación cualitativa en farmacia: aplicación en la atención farmacéutica. Revista Brasileira de Ciências Farmacêuticas. 2008;44(4):763-72.

32. Bisang R, Campi M, Cesa V. Biotecnología y desarrollo [documento de proyecto]. Washington: Comisión Económica para América Latina y el Caribe (Cepal); 2009.

33. Pogge T. Medicamentos para el mundo: impulsar la innovación sin obstaculizar el libre acceso. Sur-Revista Internacional de Derechos Humanos. 2008;5(8):120-204.

34. Gálvez GAM. La farmacoeconomía en la eficiencia de la salud pública. Rev Cubana Salud Pública. 1999;25(1):81-5.

35. López-Valcarcel BG, Cabeza MA, López CA, Díaz BJA, Álamo SF, Ortún RV Evolución de los estudios de utilización de medicamentos: del consumo a la calidad de la prescripción. Cuadernos Económicos de ICE. 2005;(67):161-189.

36. Rodríguez A. La realidad detrás de las farmacéuticas: sendas de una posmodernidad que no perdona. Rev Enferm Actual Costa Rica [internet]. 2014;(26):1-13. Disponible en: http://www.revenf.ucr.ac.cr/ farmaceutica.pdf.

37. Guzmán A, Guzmán MV. ¿Poseen capacidades de innovación las empresas farmacéuticas de América Latina?: la evidencia de Argentina, Brasil, Cuba y México. Economía: Teoría y Práctica. 2009;1(1):131-73.

38. Vallejo DBM, Torres PNS Desarrollo tecnológico del sector farmacéutico industrial asociado a procesos de transformación de materiales. Revista de la Facultad de Química Farmacéutica. 2007;14(2):59-70.

39. De Vries G, Pennings E, Block JH. Trademark or patent?: The effects of market structure, customer type and venture capital financing. Rotterdam: Department of Applied Economics, Erasmus School of Economics, Erasmus University Rotterdam; 2013. p. 1-39.

40. Barrios L. Atención farmacéutica: experiencia de cambios curriculares. Revista de la OFIL. 2010.

41. Cabral de Barros JA La publicidad y el uso adecuado de los medicamentos. Fármacos. Boletín Electrónico Latinoamericano para Fomentar el uso Adecuado de Medicamentos. 2005;8(3):1-150.

42. Velásquez G, Boulet P. Globalización y acceso a los medicamentos: implicaciones del Acuerdo de la OMC sobre los ADPIC. Primera parte: economía de la salud y medicamentos. 2a ed. Washington: Organización 
Mundial de la Salud, Departamento de Medicamentos Esenciales y Politica Farmacéutica; 2000.

43. Hardon A, Hodgkin C, Fresle D. ¿Cómo investigar el uso de medicamentos por parte de los consumidores? Ginebra: Comunidad Europea, Organización Mundial de la Salud y Universidad de Ámsterdam; 2004.

44. Puig JJ, Peiró S De la utilidad de los medicamentos al valor terapéutico añadido y a la relación coste-efectividad incremental. Rev Esp Salud Pública. 2009;83(1):59-70.

45. Consejería de Sanidad y Consumo de Madrid. Reacciones adversas a medicamentos. Boletín Informativo del Centro de Farmacovigilancia de la Comunidad de Madrid. 2005;12(1):1-8.

46. Kottow M. Comercio y salud: politicas, tratados y el mercado farmacéutico. Revista Medicina y Humanidades. 2009;(2):1-8.

47. edicamentos. Análisis Económico. 2002;17(35):185-202.

48. Escuela Nacional de Sanidad (ENS). Sobre medicamentos y farmacoeconomía. Madrid: Ministerio de Ciencia e Innovación Antonio Iñesta García-Instituto de Salud Carlos III; 2011.

49. Tolentino SM Política nacional de medicamentos en Brasil: una visión general. Revista Peruana de Medicina Experimental y Salud Pública. 2009;26(4):544-8.

50. Faus MJ. Pharmaceutical care as a response to social need. Ars Pharmaceutica. 2000;41(1):137-43. 